04.2

\title{
Локальные измерения флуктуаций радиальной скорости плазмы в токамаке ФТ-2 с помощью экваториального усиленного рассеяния
}

\author{
() А.Д. Гурченко ${ }^{1}$, Е.З. Гусаков ${ }^{1}$, А.Б. Алтухов ${ }^{1}$, В.А. Иванов ${ }^{1}$, А.В. Сидоров ${ }^{1}$, Л.А. Есипов ${ }^{1}$, T.P. Kiviniemi ${ }^{2}$, \\ Д.В. Куприенко ${ }^{1}$, S. Leerink ${ }^{2}$, С.И. Лашкул ${ }^{1}$ \\ ${ }^{1}$ Физико-технический институт им. А.Ф. Иофффе РАН, Санкт-Петербург, Россия \\ ${ }^{2}$ Department of Applied Physics, Aalto University, Espoo, Finland \\ E-mail: aleksey.gurchenko@mail.ioffe.ru
}

Поступило в Редакцию 21 апреля 2020г.

В окончательной редакции 30 апреля 2020г.

Принято к публикации 30 апреля 2020 г.

\begin{abstract}
Продемонстрирована возможность локального измерения уровня флуктуаций радиальной скорости плазмы с помощью метода экваториального усиленного рассеяния узкого пучка микроволн в верхнем гибридном резонансе во внутренних областях плазмы токамака. Выяснены ограничения предложенного метода на периферии плазмы, где растет амплитуда флуктуаций плотности и становится существенным малоугловое рассеяние микроволн на них по пути к верхнему гибридному резонансу и обратно.
\end{abstract}

Ключевые слова: плазма, токамак, флуктуации скорости, усиленное рассеяние.

DOI: 10.21883/PJTF.2020.15.49748.18348

Повышенный интерес к изучению флуктуаций радиальной скорости плазмы связан с их значительной ролью в формировании радиальных турбулентных потоков энергии и частиц в токамаках. Количественная характеризация турбулентных потоков необходима для понимания механизмов аномально высокого транспорта плазмы в токамаке и таким образом для решения одной из последних фундаментальных проблем физики высокотемпературной плазмы и управляемого термоядерного синтеза с магнитным удержанием.

В настоящее время диагностикой, позволяющей проводить локальные измерения флуктуаций радиальной скорости одновременно с флуктуациями плотности во внутренних областях плазмы, является методика зондирования пучком тяжелых ионов с применением многощелевых анализаторов энергии либо линеек детекторов [1]. Значительно более простой и дешевый метод экваториального усиленного рассеяния (УР), предложенный в [2] и используемый в настоящей работе, не предназначен для полной замены зондирования пучком тяжелых ионов, а нацелен на проведение локальных измерений величины флуктуаций радиальной скорости в более широком диапазоне длин волн в плазме исследовательских токамаков с температурой электронов в центре до $1 \mathrm{keV}$.

В применяемой на токамаке ФТ-2 (большой радиус $R=55 \mathrm{~cm}$, радиус диафрагмы $a=7.9 \mathrm{~cm}$ ) диагностике используется экваториальное микроволновое зондирование со стороны сильного магнитного поля специально сформированным (узким в полоидальном направлении) пучком волн необыкновенной поляризации с волновым вектором $\mathbf{k}_{i}$ (рис. $1, a$ ). Измеряются характеристики сигнала обратного рассеяния от мелкомасштабных флуктуаций плотности с волновым вектором $\mathbf{q}=-2 \mathbf{k}_{i}$, происходящего в узкой окрестности верхне- го гибридного резонанса (ВГР). Радиальные смещения флуктуаций плотности, вызванные движениями плазмы, приводят к появлению допплеровского частотного сдвига спектра УР. Турбулентный случайный характер радиального движения плазмы при долговременных измерениях приводит к уширению частотного спектра сигнала обратного рассеяния $2 \pi \delta f=\delta v_{r} q_{r}$, где $\delta v_{r}$ среднеквадратичное значение величины флуктуаций радиальной скорости плазмы, которое также может быть выражено через стандартное отклонение $\sigma$ от среднего значения временно́й производной полной фазы сигнала: $\delta v_{r}=2 \pi \sigma(\partial \psi / \partial t) / q_{r}$.

Измерения на ФТ-2 выполнялись в хорошо воспроизводимых омических разрядах с током $I_{p}=19 \mathrm{kA}$ и тороидальным магнитным полем $B_{0}=2.2 \mathrm{~T}$ в водородной плазме. На рис. $1, b$ показаны радиальные профили электронной плотности, а также электронной и ионной температур в исследуемом режиме, для которого предварительно было выполнено глобальное гирокинетическое (ГК) моделирование с помощью кода ELMFIRE [3].

Фаза сигнала обратного рассеяния $\psi(t)=\operatorname{arctg}(S / C)$, а также его частотный спектр мощности $P(f)$ (где $f=f_{s}-f_{i}$ - разница между частотой рассеянного сигнала и частотой зондирования) измерялись с помощью квадратурной схемы детектирования, формирующей два гомодинных сигнала $C(t)$ и $S(t)$, сдвинутых по фазе на $\pi / 2$. Эти сигналы регистрировались с тактовой частотой $50 \mathrm{MHz}$, что позволяло отслеживать эволюцию фазы с шагом по времени $20 \mathrm{~ns}$. Для измерения радиальных волновых чисел флуктуаций плотности $q_{r}$, вносящих вклад в сигнал УР, использовалась корреляционная модификация диагностики [4] и применялась схема с двухчастотным зондированием [5]. Для оценки уровня $\delta v_{r}$ в работе использовались значения $q_{r}$, соответствующие максимуму спектра сигнала $P\left(f, q_{r}\right)$. Радиальные профили зна- 

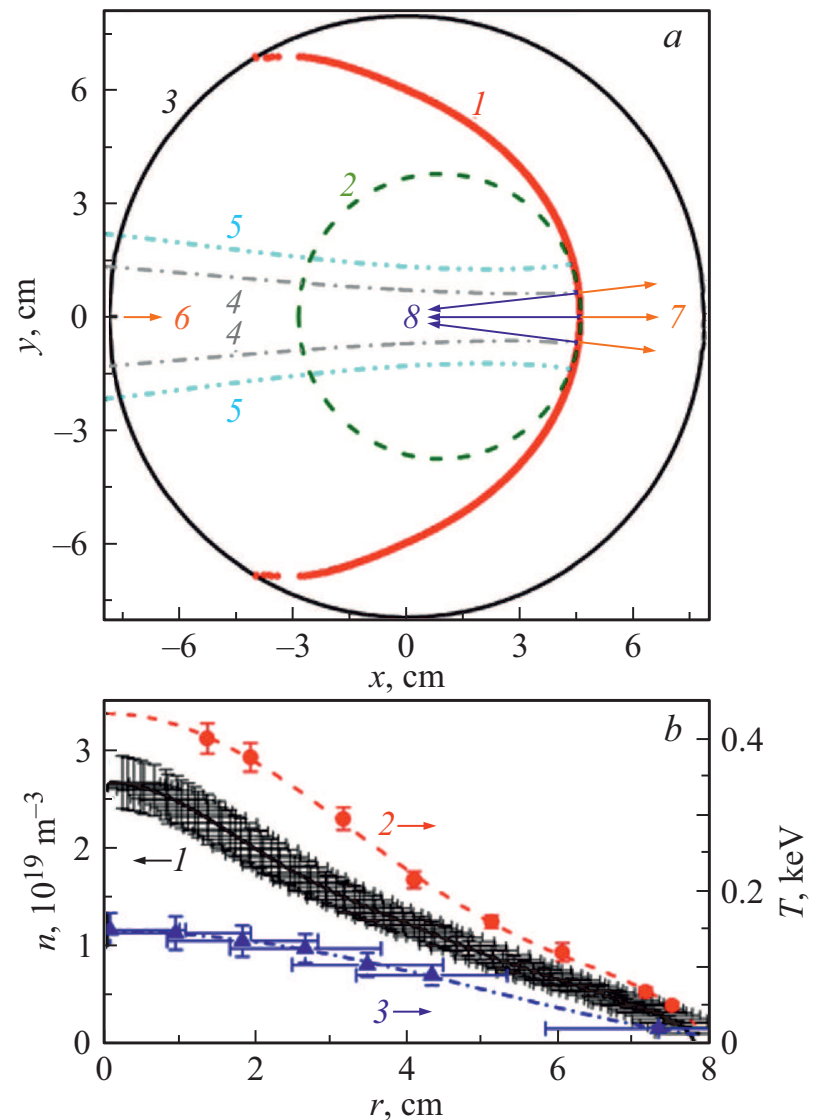

Рис. 1. $a-$ геометрия эксперимента в полоидальном сечении ФТ-2. 1 - ВГР; 2 - магнитная поверхность; 3 - диафрагма; 4 и 5 - положения на свертке диаграмм направленности зондирующей и приемной антенн, соответствующие падению уровня сигнала на $4-6$ и 5-24 dB; 6 и $7-$ волновые векторы зондирующей волны на выходе из антенны и при рассеянии в ВГР соответственно; 8 - волновые векторы флуктуаций, приводящих к обратному рассеянию. $b-$ профили плотности $(1)$, электронной (2) и ионной (3) температур.

чений $\delta v_{r}$, посчитанные по данным $\sigma(\partial \psi / \partial t)$, показаны символами на рис. 2, $a$. Приведенные на этом рисунке ошибки определения $\delta v_{r}$ учитывают конечное разрешение по радиальным волновым векторам $\Delta q_{r} \approx 8 \mathrm{~cm}^{-1}$. Кривая 1 на рис. 2, $a$ демонстрирует среднеквадратичное значение флуктуаций радиальной скорости плазмы, полученных в результате ГК-моделирования, с усреднением вдоль магнитной поверхности по всему полоидальному углу $\theta$. Следует отметить, что $\delta v_{r}$ в численном моделировании оказались неоднородны в полоидальном направлении. Соответствующие экстремальные значения показаны на рис. 2, а кривыми 2 и 3.

Помимо приведенной выше основной интерпретации уширения спектра экваториального УР также были рассмотрены два альтернативных механизма [6], не связанные с флуктуациями скорости радиального движения плазмы.

Первый связан с допплеровским уширением частотного спектра обратно рассеянного микроволнового пучка, являющегося результатом суперпозиции парциальных волн, обладающих ненулевыми полоидальными волновыми числами $q_{\theta}$. Вблизи ВГР эти волновые числа увеличиваются с ростом вертикального смещения $y$ соответствующего луча относительно экваториальной плоскости как $q_{\theta}=2 k_{\theta 0}(y)+q_{r} \cos [\alpha(y)] \equiv\left(a_{1}+a_{2} q_{r}\right) y$ [7]. Полоидальное волновое число $k_{\theta 0}$ зондирующего луча вблизи ВГР (до его резонансного возрастания) и угол $\alpha$ между нормалью к поверхности ВГР (совпадающей с локальным направлением луча) и касательной к магнитной поверхности могут быть вычислены с помощью метода лучевых траекторий. Сравнение экспериментальных спектров с модельным выражением для формы спектра $P_{m}(f) \propto \exp \left[-a_{3}\left(2 \pi f / v_{\theta}\right)^{2}\left(a_{1}+a_{2} q_{r}\right)^{-2}\right], \quad$ полученным из полоидального распределения интенсивности волнового пучка на поверхности ВГР $P_{a}^{2}=\exp \left[-a_{3} y^{2}\right]$ путем представления вертикального смещения $y$ через $q_{\theta}$ и
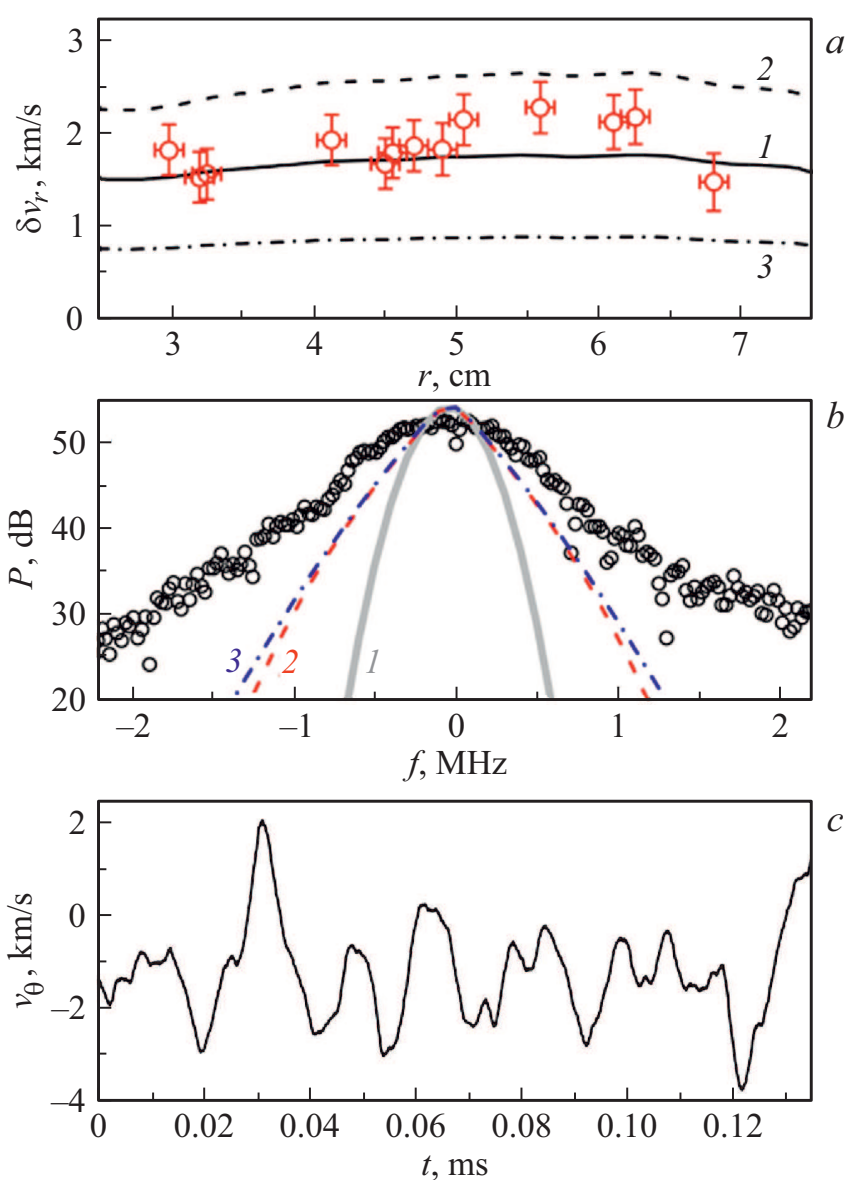

Рис. 2. $a-$ профили уровня флуктуаций радиальной скорости плазмы, оцененные на основе фазовых измерений сигнала экваториального УР (символы) и полученные в результате ГК-моделирования (линии): после усреднения вдоль всей магнитной поверхности (1) либо вблизи экватора со стороны слабого (2) и сильного (3) магнитного поля. $b-$ спектр УР (символы) для $r=5 \mathrm{~cm}$ и $q_{r}=85 \mathrm{~cm}^{-1}$, а также модельные спектры (линии): $1-P_{m}$ для $\left\langle v_{\theta}\right\rangle=-1.24 \mathrm{~km} / \mathrm{s} \quad\left(a_{1}=12.38 \mathrm{~cm}^{-2}\right.$, $\left.a_{2}=0.083 \mathrm{~cm}^{-1}, a_{3}=2.9\right), 2-P_{G}$ при усреднении за время $\Delta t=0.07 \mathrm{~ms}, 3-P_{G}$ при $\Delta t=0.14 \mathrm{~ms} . c-$ эволюция $v_{\theta}$ для $r=5 \mathrm{~cm}$. 


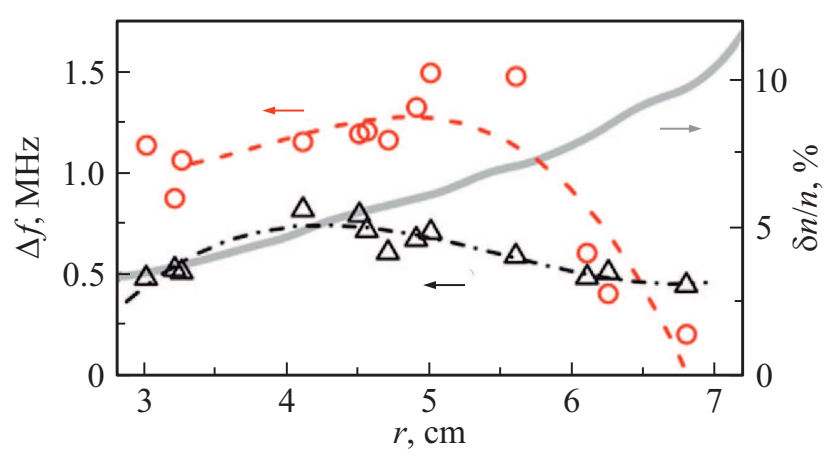

Рис. 3. Ширина измеренного спектра УР по уровню $1 / e$ (кружки), оценка уширения спектра на пути к ВГР и обратно в рамках турбулентной теории (треугольники) и относительный уровень флуктуаций плотности (сплошная линия).

использования его связи с частотой $\left(v_{\theta}=2 \pi f / q_{\theta}\right)$, показано на рис. $2, b$. Следует отметить, что радиальный профиль средней полоидальной скорости плазмы $\left\langle v_{\theta}\right\rangle$ в исследуемых режимах был ранее детально изучен [3]. Измеренные абсолютные значения $\left\langle v_{\theta}\right\rangle$ уменьшались от $1.45 \pm 0.25 \mathrm{~km} / \mathrm{s}$ при $r / a=0.47$ до $0.7 \pm 0.4 \mathrm{~km} / \mathrm{s}$ на краю плазмы при $r / a=0.85$. Сравнение относительно широкого экспериментального спектра (символы на рис. 2,b) с приведенным выше выражением (кривая 1) показывает, что в рамках рассматриваемой модели спектр невозможно описать из-за узости используемого для зондирования микроволнового пучка и малого значения полоидальной скорости плазмы $\left\langle v_{\theta}\right\rangle$, измеренной в этом режиме.

Важной особенностью полоидального вращения плазмы в рассматриваемом разряде является наличие области $(0.5<r / a<0.7)$ с интенсивными колебаниями полоидального вращения, вызванными геодезической акустической модой (ГАМ), с амплитудами, превышающими $\left\langle v_{\theta}\right\rangle$ (рис. 2,c). В нашем анализе фактическое присутствие ГАМ учитывалось путем подстановки временно́й эволюции значений полоидальной скорости $v_{\theta}(t)$ в указанное выше модельное выражение для формы спектра и осуществления его интегрирования по времени $P_{G}=\int P_{m}\left[f, v_{\theta}(t)\right] d t / \Delta t$ в течение нескольких периодов ГАМ. Модельные спектры, полученные таким образом, показаны на рис. $2, b$ линиями 2 и 3 . Как видно, влияние переменной $v_{\theta}(t)$ приводит к росту крыльев спектра, однако количественный анализ демонстрирует, что вклад ГАМ даже в точке их максимальной интенсивности оказывается недостаточным для описания широкого экспериментального спектра.

Вторым механизмом, приводящим к уширению спектра сигнала УР, является многократное малоугловое рассеяние зондирующих и рассеянных в ВГР волн на пути к поверхности ВГР и обратно. Согласно теории распространения электромагнитной волны в области ВГР в турбулентной плазме [8], ширина ее спектра на уровне $\max (P) / e$ равна $\Delta f=q_{r}^{2}(\delta n / n) \sqrt{L_{n} l_{\delta n}\left\langle f_{0}^{2}\right\rangle} /\left(8 \omega_{c e} / c\right)$, где
$L_{n}$ - масштаб неоднородности профиля плотности, $\omega_{c e}$ - электронно-циклотронная частота в ВГР, $\delta n / n-$ относительный уровень флуктуаций плотности, $\sqrt{\left\langle f_{0}^{2}\right\rangle}=0.24 \pm 0.04 \mathrm{MHz}$ - ширина их частотного спектра (оба параметра оцениваются в рамках ГК-моделирования), $l_{\delta n} \approx 0.5 \mathrm{~cm}-$ корреляционная длина флуктуаций плотности, измеренная экспериментально [9].

Сравнение ширины экспериментальных спектров с теоретическим предсказанием показано на рис. 3. На периферии плазмы $(r / a>0.75)$, где $\delta n / n>0.08$, рассмотренный механизм многократного малоуглового рассеяния достаточно силен, чтобы отвечать за уширение всего спектра. Во внутренних же областях его влияние меньше, так что доминирующий механизм формирования спектра связан с колебаниями радиальной скорости плазмы, которые увлекают мелкомасштабные флуктуации плотности.

В заключение следует отметить, что в работе не рассматривается одновременное проявление нескольких механизмов уширения спектров, а лишь выявляются условия, когда тот или иной механизм оказывается доминирующим. Основным результатом работы является экспериментальная демонстрация возможности локального измерения уровня флуктуаций радиальной скорости плазмы в токамаке, а также условий, при которых предложенный метод перестает работать.

\section{Финансирование работы}

Работа выполнена при поддержке Российского научного фонда (проект № 17-12-01110). Численное моделирование поддержано грантами Академии Финляндии 316088 и 330342. Авторы благодарны CSC - IT Center for Science за щедрое распределение вычислительных ресурсов для этой работы. Эксплуатация токамака ФТ-2 и стандартных диагностик разряда выполнялась в рамках государственного контракта ФТИ им. А.Ф. Иоффе 0040-2014-0023.

\section{Конфоликт интересов}

Авторы заявляют, что у них нет конфликта интересов.

\section{Список литературы}

[1] Melnikov A.V., Krupnik L.I., Eliseev L.G., Barcala J.M., Bravo A., Chmyga A.A., Deshko G.N., Drabinskij M.A., Hidalgo C., Khabanov P.O., Khrebtov S.M., Kharchev N.K., Komarov A.D., Kozachek A.S., Lopez J., Lysenko S.E., Martin G., Molinero A., de Pablos J.L., Soleto A., Ufimtsev M.V., Zenin V.N., Zhezhera A.I., T-10 Team and TJ-II Team // Nucl. Fusion. 2017. V. 57. P. 072004.

[2] Гурченко А.Д., Гусаков Е.3. // Письма в ЖТФ. 2018. Т. 44. B. 8. C. $49-56$.

[3] Niskala P., Gurchenko A.D., Gusakov E.Z., Altukhov A.B., Esipov L.A., Chôné L., Kiviniemi T.P., Leerink S. // Nucl. Fusion. 2018. V. 58. P. 112006. 
[4] Gusakov E.Z., Kaganskaya N.M., Kraemer M., Selenin V.L. // Plasma Phys. Control. Fusion. 2000. V. 42. P. 1033-1049.

[5] Gusakov E.Z., Gurchenko A.D., Altukhov A.B., Bulanin V.V., Esipov L.A., Kantor M.Yu., Kouprienko D.V., Lashkul S.I., Petrov A.V., Stepanov A.Yu. // Plasma Phys. Control. Fusion. 2006. V. 48. P. B443-B451.

[6] Gurchenko A.D., Gusakov E.Z., Larionov M.M., Novik K.M., Savel'ev A.N., Selenin V.L., Stepanov A.Yu. // Plasma Phys. Rep. 2004. V. 30. P. 807-815.

[7] Bulyiginskiy D.G., Gurchenko A.D., Gusakov E.Z., Korkin V.V., Larionov M.M., Novik K.M., Petrov Yu.V., Popov A.Yu., Saveliev A.N., Selenin V.L., Stepanov A.Yu. // Phys. Plasmas. 2001. V. 8. P. 2224-2231.

[8] Gusakov E.Z., Surkov A.V. // Plasma Phys. Rep. 2002. V. 28. P. 827-836.

[9] Altukhov A., Gurchenko A.D., Gusakov E.Z., Esipov L.A., Irzak M.A., Kantor M.Yu., Kouprienko D.V., Lashkul S.I., Leerink S., Niskala P., Stepanov A.Yu., Teplova N.V. // Plasma Phys. Control. Fusion. 2016. V. 58. P. 105004. 